\title{
Análise de existência e comportamento de onda viajante para equações de Euler com relaxação
}

\author{
Eduardo Abreu, $\quad \underline{\text { Abel Alvarez Bustos, }}$, \\ Instituto de Matemática, Estatística e Computação Científica, IMECC, UNICAMP, \\ 13083-859, Campinas, SP \\ E-mail: eabreu@ime.unicamp.br, abelalv@ime.unicamp.br, \\ Wanderson José Lambert \\ UFRRJ - Departamento de Matemática \\ Campus Seropédica \\ 23890-000, Seropédica, RJ \\ E-mail: wjlambert@ufrrj.br.
}

Resumo: Neste trabalho estudamos um sistema de equações de Euler com relaxação generalizada. O sistema modelo de equações de Euler admite ainda um desacoplamento para sua solução. Inicialmente resolvemos o sistema como um problema de Riemann e aplicamos uma regularização a fim de tornar a solução de classe $\mathcal{C}^{1}$. O sistema geral resultante deste procedimento é resolvido via técnica de curvas características. Mais do que isso, introduzindo um parâmetro $\beta$ com base na modelagem física, estudamos condições para a existência de onda viajante, além de analisar a relação entre as escalas físicas da difusão e de relaxação.

Palavras-chave: Sistema incompressivel de equações de Euler com relaxação generalizada, solução de Riemann, onda viajante e perfil viscoso.

\section{Introdução}

Estamos interessados em analisar a existência de choques com perfil viscoso para o seguinte sistema de equações de Euler com relaxação generalizada, via um parâmetro de velocidade $\beta$,

$$
\frac{\partial}{\partial t}\left[\begin{array}{c}
\rho \\
m \\
E
\end{array}\right]+\frac{\partial}{\partial x}\left[\begin{array}{c}
m \\
\frac{m^{2}}{\rho}+a^{2} \rho \\
(\beta E+p) u
\end{array}\right]=\frac{1}{\epsilon}\left[\begin{array}{c}
0 \\
0 \\
(\bar{E}(\rho, \rho u)-E)
\end{array}\right],
$$

no qual $\rho$ é a densidade, $m$ o momento linear, $p$ a pressão e $E$ a energia. $\beta$ é um parâmetro que introduzimos para estudar a existência, ou não, de onda viajante dentro da solução, mas que tem uma justificativa física para o modelo em questão [6]. A quantidade $\bar{E}(\rho, \rho u)$ é vista como a energia necessária para trazermos a temperatura $T$ para uma temperatura de referência $\bar{T}$ sem nenhuma mudança no momento ou energia do gás. Considerando a equação de estado para gases ideais politrópicos, a pressão pode ser escrita como (veja [4]) $p=R \rho \bar{T}=a^{2} \rho$, no qual $a \sqrt{R \bar{T}}$ é a velocidade do som. A energia de referência é escrita como $\bar{E}(\rho, \rho u)=\frac{a^{2} \rho}{\gamma-1}+\frac{1}{2} \rho u^{2}$. O termo $\epsilon$ representa o chamado tempo de relaxação, que está associado ao tempo em que a energia $E$ está longe da energia de equilíbrio $\bar{E}(\rho, \rho u)$. Do ponto de vista prático, apesar desse tempo ser bastante pequeno, ele é diferente de zero. Como estamos interessados em analisar a existência de ondas viajantes, e perfis viscosos, é útil que resolvamos o problema de Riemann associado

${ }^{*}$ Bolsista de doutorado FAPESP - Processo FAPESP 11/23628-0 
a este sistema, veja [2]. O problema de Riemann em questão consiste de (1) em conjunto das condições iniciais especiais:

$$
\begin{cases}\left(m_{l}, \rho_{l}, E_{l}\right), & \text { se } x<0 \\ \left(m_{r}, \rho_{r}, E_{r}\right), & \text { se } x>0\end{cases}
$$

ou seja, condições que são constantes por partes.

Na Seção 2 descrevemos a solução do problema de Riemann (1)-(2), bem como uma regularização necessária para obtermos uma aproximação global da solução. Em seguida, na Seção 3, analisamos as condições sobre o parâmetro $\beta$ para a existência de onda viajante.

\section{Solução do Problema de Riemann}

O sistema (1) tem uma peculiaridade bastante interessante. As duas primeiras equações para a densidade $\rho$ e o momento $m$ não dependem da energia. Dessa forma, podemos desacoplá-lo inicialmente em um sistema (hiperbólico) $2 \times 2$ de leis de conservação, como segue:

$$
\frac{\partial}{\partial t}\left[\begin{array}{c}
\rho \\
m
\end{array}\right]+\frac{\partial}{\partial x}\left[\begin{array}{c}
m \\
m^{2} / \rho+a^{2} \rho
\end{array}\right]=\left[\begin{array}{l}
0 \\
0
\end{array}\right], \quad a \equiv \text { constante, } \quad t>0, \quad-\infty<x<\infty
$$

e com dados iniciais de (2), tais que,

$$
\rho(x, 0)=\left\{\begin{array}{ll}
\rho_{l}, & \text { se } x \leq 0, \\
\rho_{r}, & \text { se } x>0,
\end{array} \quad m(x, 0)= \begin{cases}m_{l}, & \text { se } x \leq 0, \\
m_{r}, & \text { se } x>0 .\end{cases}\right.
$$

No presente trabalho, vamos analisar em detalhes o caso apenas em que $\rho_{l}>\rho_{r}$ e $m_{l}>m_{r}$. Entretanto, a análise para todos os demais possíveis casos segue diretamente da mesma técnica aqui desenvolvida. É fácil de ver que (3) é estritamente hiperbólico, com autovalores distintos $\lambda_{1}=m / \rho-a$ e $\lambda_{1}=m / \rho+a$. O subsistema (3)-(4) tem como solução (ver, e.g., [2]),

$$
\rho=\left\{\begin{array}{l}
\rho_{l}, \quad x<t \lambda_{1}\left(\rho_{l}, m_{l}\right), \\
\rho\left(\frac{x}{t}\right), \quad t \lambda_{1}\left(\rho_{l}, m_{l}\right) \leq x<t \lambda_{1}\left(\rho_{m}, m_{m}\right), \\
\rho_{m}, \quad t \lambda_{2}\left(\rho_{m}, m_{m}\right) \leq x<t s, \\
\rho_{r}, \quad t s \leq x,
\end{array} \quad m=\left\{\begin{array}{l}
m_{l}, \quad x<t \lambda\left(\rho_{l}, m_{l}\right), \\
m\left(\frac{x}{t}\right), \quad t \lambda\left(\rho_{l}, m_{l}\right) \leq x<t \lambda\left(\rho_{m}, m_{m}\right), \\
m_{m}, \quad t \lambda\left(\rho_{m}, m_{m}\right) \leq x<t s, \\
m_{r}, \quad t s \leq x,
\end{array}\right.\right.
$$

no qual $\left(\rho_{m}, m_{m}\right)$ é um estado intermediário, $s=\frac{m_{r}-m_{l}}{\rho_{l}-\rho_{r}}$ é a velocidade do choque e

$$
\rho\left(\frac{x}{t}\right)=\rho_{l} \exp \left\{-\left(\frac{x}{t}-\eta_{l}\right) / a\right\}, \quad m\left(\frac{x}{t}\right)=\rho_{l}\left(\frac{x}{t}+a\right) \exp \left\{-\left(\frac{x}{t}-\eta_{l}\right) / a\right\} .
$$

A solução (5) de (3)-(4) consiste de um estado constante $\left(\rho_{l}, m_{l}\right)$, seguida por uma rarefação $(\rho(x / t), m(x / t))$, depois por um estado constante $\left(\rho_{m}, m_{m}\right)$, seguido por um choque de velocidade $s$ conectando $\left(\rho_{m}, m_{m}\right)$ a $\left(\rho_{r}, m_{r}\right)$; veja a Figura 1. A solução dada por (5), torna-se apenas uma função de $x$ e $t$ no plano $x-t$. Como estratégia para encontrar a solução podemos utilizar a técnica das características para calcular a solução da $E=E(x, t)$ (ver, e.g., [3]). Entretanto, para podermos aplicar a técnica das curvas características, precisamos resolver um conjunto de equações diferenciais ordinárias para fluxos contínuos, o que não é o caso pois tanto $m$ quanto $\rho$ apresentam descontinuidades ao longo do eixo $x$. Por outro lado, podemos supor por um momento que $\rho=\rho(x, t)$ e $m=m(x, t)$ são suficientemente suaves. Assim, por meio da equação para a energia em (1) podemos escrever:

$$
E_{t}+E_{x} \frac{\beta m}{p}=-E\left(\left[\frac{m}{p}\right]_{x}+\frac{1}{\epsilon}\right)+\frac{1}{\epsilon}\left(\frac{a^{2} p}{\gamma-1}+\frac{1}{2} \frac{m^{2}}{p}\right)-[m]_{x},
$$



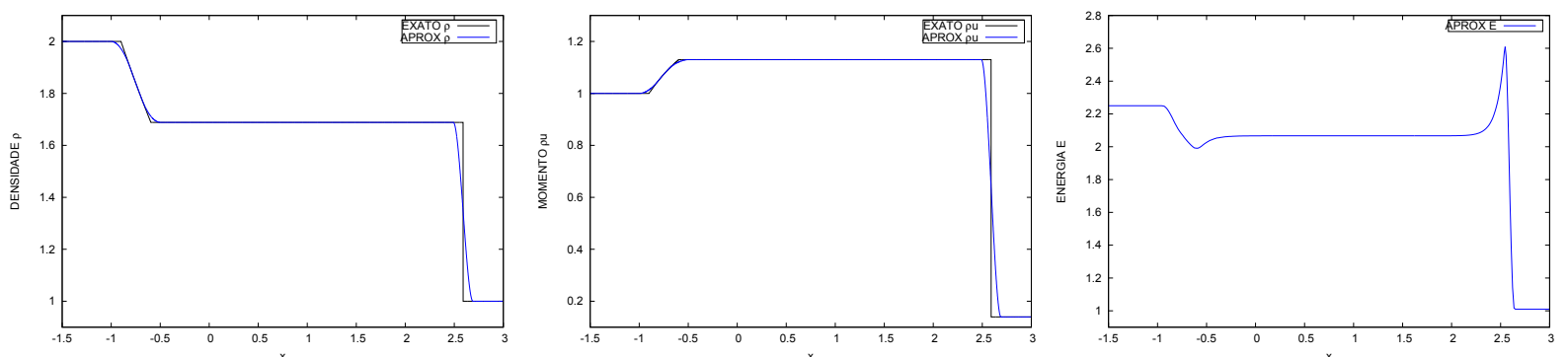

Figura 1: Soluções para $\rho$ (esquerda) e $m$ (centro), e suas respectivas aproximações. À direita encontra-se a solução para a energia $E$. Note que ocorre um pico justamente nesta solução. Usamos $\left(\rho_{l}, m_{l}\right)=(2,1)$ e $\left(\rho_{r}, m_{r}\right)=(1,0.13)$ e tempo de impressão da solução $t=1.8$.

com $E(x, 0)=E_{l}$, se $x<0$ e $E(x, 0)=E_{l}$, se $x>0$. A solução sobre as características fica,

$$
\frac{d x}{d t}=\frac{\beta m}{p}, \quad \frac{d E}{d t}=-E\left(\left[\frac{m}{p}\right]_{x}+\frac{1}{\epsilon}\right)+\frac{1}{\epsilon}\left(\frac{a^{2} p}{\gamma-1}+\frac{1}{2} \frac{m^{2}}{\rho_{\tau}}\right)-[m]_{x},
$$

com condições iniciais,

$$
x(0)=x_{i}, \quad \text { com } \quad E(x, 0)=E_{l}, \quad \text { se } \quad x<0 \quad E(x, 0)=E_{l}, \quad \text { se } \quad x>0 .
$$

Como a solução 5 exibe descontinuidades nos choques e não é diferenciável nos pontos iniciais e finais das rarefações, precisamos regularizar as soluções $\rho$ e $m$ a fim de conseguirmos resolver o sistema (8)-(9). Vamos encontrar funções $\rho_{\tau}, m_{\tau} \in C^{1}(\mathcal{R})$, dependendo de um parâmetro $\tau$ de modo que se $\tau \rightarrow 0$ então $\rho_{\tau}, m_{\tau}$ convergem para $\rho$ e $m$ dados por (5). Essa convergência é considerada na norma $L^{p}$ para qualquer $1 \leq p$.

Para buscar tais funções, procuramos polinômios que são suaves na vizinhança de $\rho$, os quais dependem de $\tau$. Para o ponto $t \lambda_{1}\left(\rho_{l}, m_{l}\right)$ é necessário um polinômio de terceiro grau da forma $p(z)=a_{3} z^{3}+a_{2} z^{2}+a_{1} z+a_{0}, z \in\left[t \lambda_{1}\left(\rho_{l}, m_{l}\right)-\tau, t \lambda_{1}\left(\rho_{l}, m_{l}\right)+\tau\right]$, com as seguintes propriedades, para $\lambda_{1, l}=\lambda_{1}\left(\rho_{l}, m_{l}\right)$ e $\lambda_{1, m}=\lambda_{1}\left(\rho_{m}, m_{m}\right)$, talque:

$$
p_{1}\left(t \lambda_{1, l}-\tau, t\right)=\rho_{l}, \quad p_{1}^{\prime}\left(t \lambda_{1, l}-\tau, t\right)=0, \quad p_{1}\left(t \lambda_{1, m}+\tau, t\right)=\rho_{m}, \quad p_{1}^{\prime}\left(t \lambda_{1, m}+\tau, t\right)=\rho^{\prime}(x / t) .
$$

Para obter a solução de (10), resolve-se um sistema linear $4 \times 4$ [1] para descobrir o único polinômio com coeficientes $a_{0}, a_{1}, a_{2}$ e $a_{3}$. Por meio de cálculos similares conseguimos regularizar o problema e encontrar soluções aproximadas, que dependem do parâmetro $\tau$ (ver [1]):

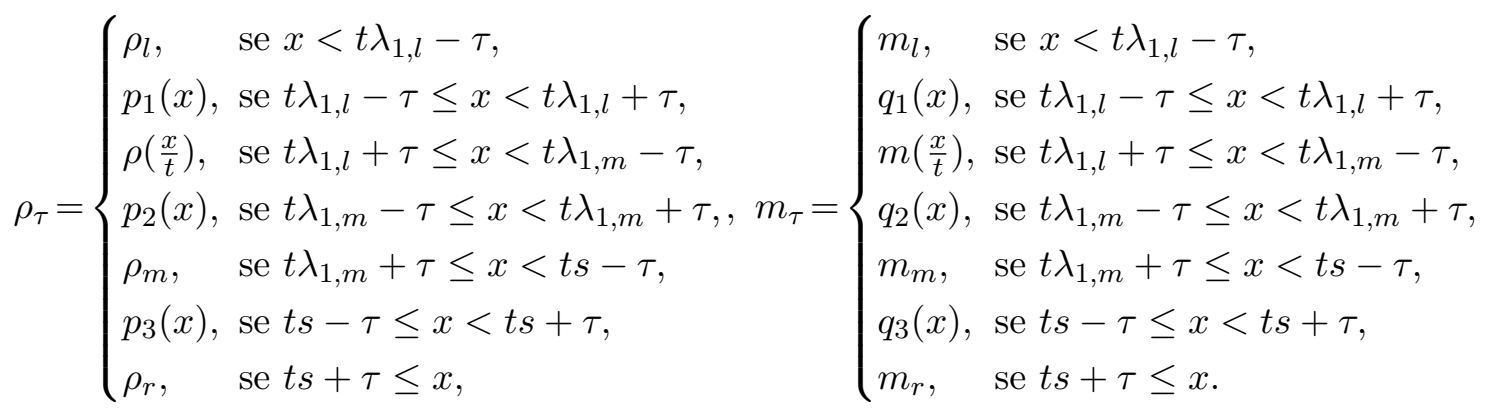

Na Figura 1, descrevemos a solução e o formato das regularizações da solução, Substituímos (11) dentro de (8)-(9) e obtemos, como solução

$$
E(\tau)=\exp (-\tau / \epsilon+1) E_{0}+\exp (-\tau / \epsilon) \int_{0}^{\tau} \exp (s / \epsilon)\left[\frac{1}{\epsilon}\left(\beta \rho+\frac{m^{2}}{2 \rho}\right)-m_{x}\right] d s,
$$

onde $\eta=x / t, \rho(\eta)=\rho_{l} \exp \left\{-\left(\eta-\eta_{l}\right) / a\right\}$ and $m(\eta)=\rho_{l}(\eta+a) \exp \left\{-\left(\eta-\eta_{l}\right) / a\right\}$. Na Figura 1, exibimos a solução de $E$ para um valor de $\beta=1$. Apesar da mudança da solução induzida 

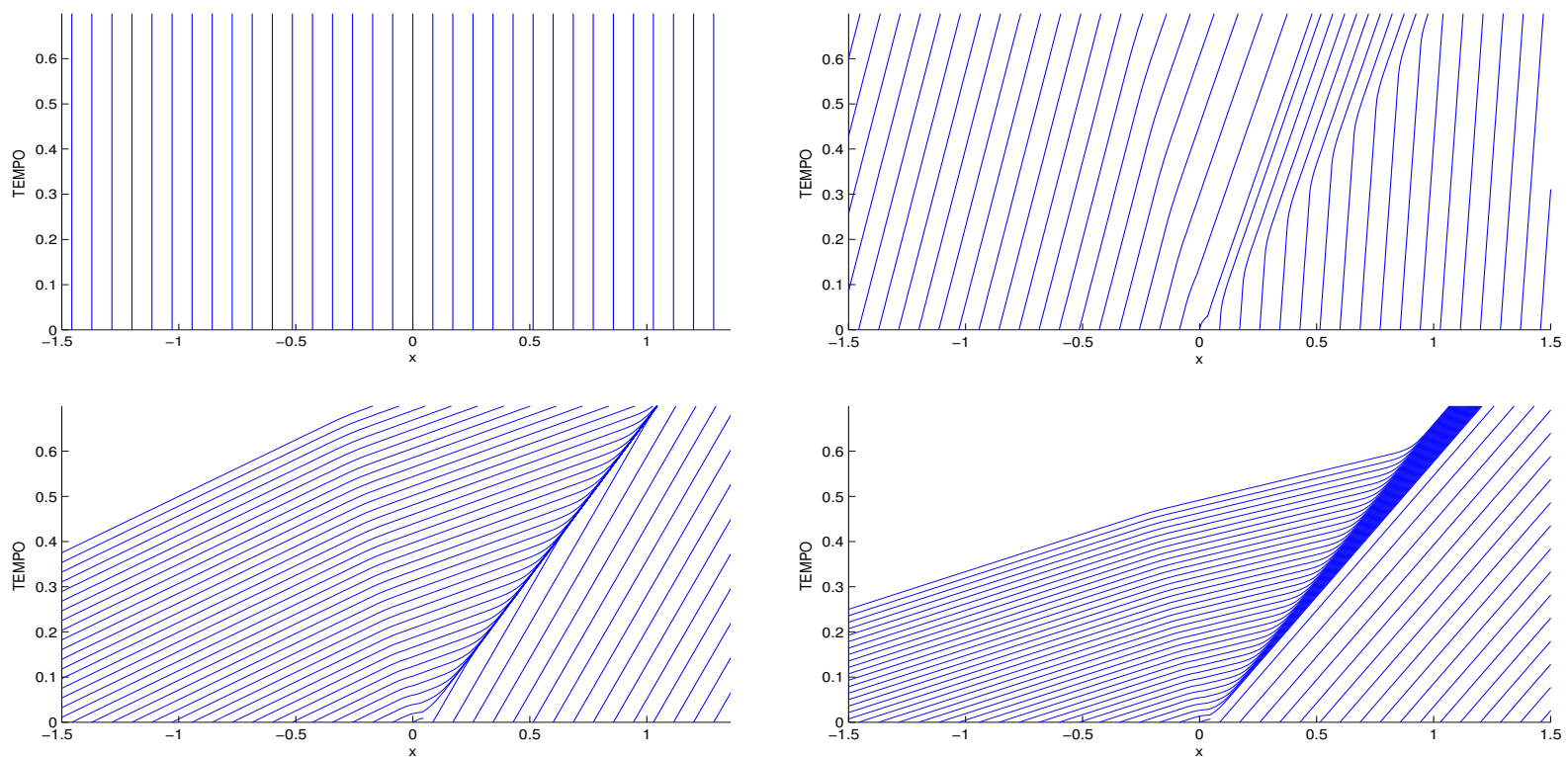

Figura 2: Esquerda Acima: Curvas características paralelas para $\beta=0$. Direita acima: $(\beta=1)$ Note que há uma região parecida como um leque de rarefação, após a curva saindo de $x=0$. A curva de choque de velocidade $s$ do sistema $(\rho, m)$ irá atravessar as características influenciando a solução da energia $E$ : a solução não exibirá perfil viscoso neste caso. Esquerda Abaixo: $(\beta=8)$ Observe que as curvas características "parecem" todas colidir (o que não acontece, de fato, devido à unicidade da solução para as curvas características no plano $x-t$ ): a solução exibe perfil viscoso. Aqui a curva de choque atravessa a solução das curvas características fazendo a solução se acumular em torno do choque $s$. Direita abaixo: $(\beta=12)$ Comportamento parecido com o caso anterior. Entretanto, as curvas se acumulam mais rapidamente: aqui a solução também exibe um perfil viscoso.

pela variação do parametro $\beta$, como veremos na próxima seção, temos que qualitativamente visualmente a solução não se altera substancialmente. Neste contexto, uma análise bastante relevante é considerar as curvas características para diferentes valores de $\beta$, o que fazemos na Figura 2. Note que quando os valores de $\beta$ aumentam, as curvas de onda vão se inclinando até quase se colapsarem no plano $x-t$.

\section{Condições para a existência da onda viajante}

A solução dada por (11)-(12) é uma solução que reflete o comportamento qualitativo de (1)-(2). É verdadeiramente incomum a existência de uma variação tão abrupta na solução. De fato, isso ocorre devido ao fato de existir variações abruptas da variável $m$; note que sua derivada aparece de forma explícita no lado direito de (8). Entretanto, um questionamento bastante natural é: qual é a natureza desse onda ? Observamos por meio de várias simulações numéricas [1], que tal onda não modifica seu perfil ao longo da evolução para tempos longos (comportamento assintótico). Dessa forma, é igualmente natural questionar seria esta uma onda viajante (ver, e.g., $[2])$.

A onda viajante é uma das inúmeras técnicas utilizadas para selecionar os choques que são fisicamente viáveis $[2,5]$. A existência da onda viajante é uma das principais ferramentas de análise em problemas envolvendo leis de conservação para dizer se um choque, de fato, provém de um sistema físico. Dessa forma é natural utilizarmos tal técnica para analisarmos sob quais condições a onda contendo um pico $E$ é uma onda viajante.

A maioria de sistemas modelando fenômenos físicos exibe difusão devido a diferentes fatores influenciando o movimento dos fluidos. Para tanto, nós admitimos que o sistema possui uma difusão de ordem $\eta$, que é justificada pela natureza física do modelo de Euler com friccão e 
gravidade com uma equação de Darcy parabólica em meios porosos [6]. A difusão irá influenciar, principalmente, as variáveis $\rho$ e $m$ nas duas primeiras equações. A equação para energia possui um termo de relaxação que sob as hipóteses de condições sub-características, veja [5, 4], funciona como um termo de dissipação. De fato, o sistema (1) satisfaz as condições sub-características. Dessa forma o sistema (1) é escrito como:

$$
\begin{aligned}
\frac{\partial}{\partial t} \rho+\frac{\partial}{\partial x} m & =\eta \frac{\partial^{2}}{\partial x^{2}} \rho, \\
\frac{\partial}{\partial t} m+\frac{\partial}{\partial x}\left(\frac{m^{2}}{\rho}+a^{2} \rho\right) & =\eta \frac{\partial^{2}}{\partial x^{2}} m, \\
\frac{\partial}{\partial t} E+\frac{\partial}{\partial x}\left[\beta E \frac{m}{\rho}+a \frac{m^{2}}{\rho}\right] & =\frac{1}{\epsilon}\left(\frac{a^{2} \rho}{\gamma-1}+\frac{1}{2} \frac{m^{2}}{\rho}-E\right) .
\end{aligned}
$$

A pergunta relevante aqui é: sob quais condições, o choque conectando $\left(\rho_{m}, m_{m}\right)$ e $\left(\rho_{r}, m_{r}\right)$, comporta-se como uma onda viajante? Ou seja, para uma variável $U^{\eta}=(\rho, m, E)$, a solução de (13) pode ser escrita em um sistema de coordenadas viajantes $\frac{x-s t}{\eta}$, onde $U^{\eta}=U\left(\frac{x-s t}{\eta}\right)$, tal que $s=\frac{m_{r}-m_{m}}{\rho_{r}-\rho_{m}}$ é a velocidade do choque. Para a nova variável $\eta$ a existência do perfil viscoso, está associada a existência de uma onda conectando o estado $\left(\rho_{m}, m_{m}, E_{m}\right)$ quando $\eta \longrightarrow-\infty$ até o estado $\left(\rho_{r}, m_{r}, E_{r}\right)$ quando $\eta \longrightarrow+\infty$.

Substituindo $U^{\eta}=(\rho, m, E)$ no sistema (13) e aplicando a regra da cadeia, obtemos um sistema de equações diferenciais ordinárias de segunda ordem:

$$
\begin{aligned}
\frac{-s}{\eta} \frac{d p}{d \xi}+\frac{1}{\eta} \frac{d m}{d \xi} & =\frac{\eta}{\eta^{2}} \frac{d^{2} \rho}{d \xi^{2}} \\
\frac{-s}{\eta} \frac{d p}{d \xi}+\frac{1}{\eta} \frac{d m}{d \xi}\left(\frac{m^{2}}{\rho}+a^{2} \rho\right) & =\frac{\eta}{\eta^{2}} \frac{d^{2} m}{d \xi^{2}} \\
-s \frac{d E}{d \xi}+\frac{d}{d \xi}\left[\beta E \frac{m}{\rho}+a \frac{m^{2}}{\rho}\right] & =\frac{\eta}{\epsilon}\left(\frac{a^{2} \rho}{\gamma-1}+\frac{1}{2} \frac{m^{2}}{\rho}-E\right) .
\end{aligned}
$$

Integrando no intervalo $(-\infty, \xi)$ as duas primeiras equações (14) e usando o fato que $\frac{d}{d \xi} \rho$ e $\frac{d}{d m} \rho$ se anulam (pois são equilíbrios) em $-\infty$, obtemos o sistema de primeira ordem conectando equilíbrios:

$$
\begin{aligned}
\frac{d}{d \xi} \rho & =-s \rho+m+s \rho^{-}-m^{-} \\
\frac{d}{d \xi} m & =-s m+\frac{m^{2}}{\rho}+a^{2} \rho+s m^{-}-\left(\frac{m^{2}}{\rho}\right)^{-}-a^{2} \rho^{-}, \\
\frac{d}{d \xi} E & =\frac{1}{\left(-s+\beta \frac{m}{\rho}\right)}\left[-E\left(\frac{\eta}{\epsilon}+\beta \frac{d}{d \xi}\left(\frac{m}{\rho}\right)\right)-a \frac{d}{d \xi}\left(\frac{m^{2}}{\rho}\right)+\frac{\eta}{\epsilon}\left(\frac{a^{2} \rho}{\gamma-1}+\frac{1}{2} \frac{m^{2}}{\rho}\right)\right] .
\end{aligned}
$$

Escrevendo $V=(\rho, m, E)^{T}$ e $F(V)=\left(F_{1}(V), F_{2}(V), F_{2}(V)\right)^{T}$, com os $F_{i}(V)$ em cada um dos 3 lados direitos de (15). O sistema (15) é escrito na forma vetorial como $\frac{d V}{d t}=F(V)$. Note que os pontos $\left(p_{m}, m_{m}, E_{m}\right)$ e $\left(p_{r}, m_{r}, E_{r}\right)$ onde $E_{m}=\frac{a^{2} \rho_{m}}{\gamma-1}+\frac{1}{2} \frac{m_{m}^{2}}{\rho_{m}}$ e $E_{r}=\frac{a^{2} \rho_{r}}{\gamma-1}+\frac{1}{2} \frac{m_{r}^{2}}{\rho_{m} r}$, respectivamente, são pontos de equilíbrio, o qual nos motiva fazer um estudo em torno desses equilíbrios. Para tanto, linearizamos o sistema $\frac{d V}{d t}=F(V)$. Os autovalores associados a esta linearização são $\lambda_{1}=-s+\frac{m^{-}}{\rho^{-}}-a, \lambda_{2}=-s+\frac{m^{-}}{\rho^{-}}+a$ e $\lambda_{3}=\frac{\frac{\eta}{\epsilon}+\beta \frac{d}{d \xi}\left(\frac{m}{\rho}\right)}{s-\beta \frac{m}{\rho}}$. Além disso, observe que os dois primeiros autovalores não dependem de $E$. Então podemos desacoplar o sistema (15) 

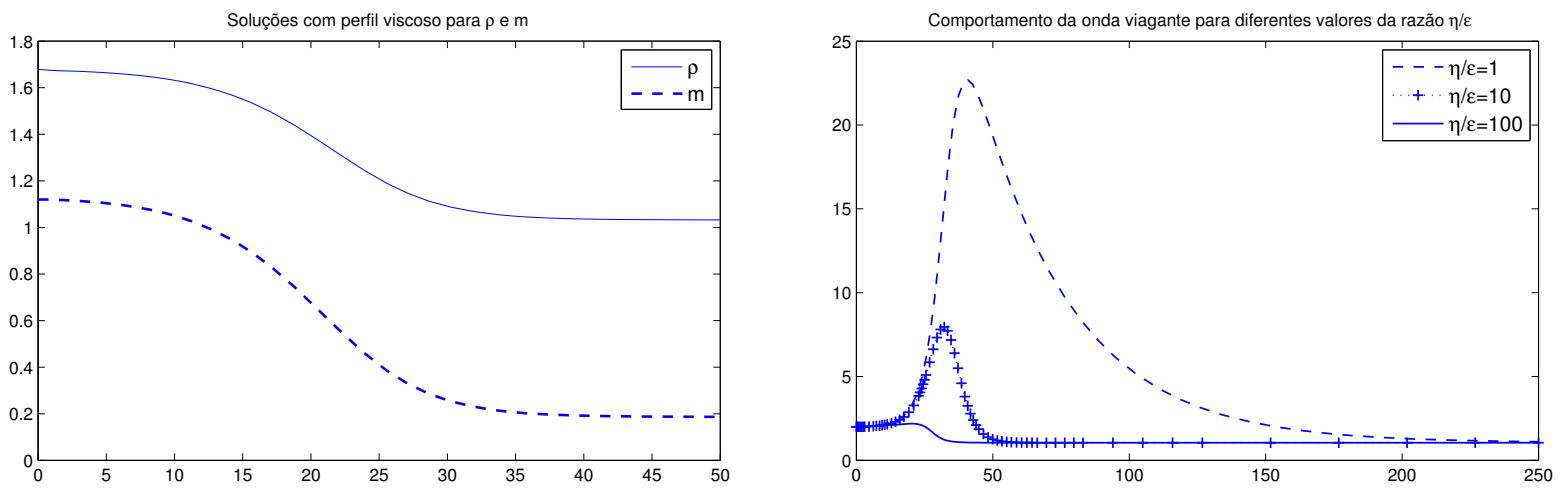

Figura 3: Esquerda. Perfil viscoso para as variáveis $(\rho, m)$. Os estados dos equilíbrios sendo conectados são $\left(\rho_{m}=1.68, m_{m}=1.12\right)$ e $\left(\rho_{r}=1, m_{r}=0.13\right)$. Direita. Solução da órbita conectando os equilíbrios $E_{m}$ e $E_{r}$ para diferentes relações entre $\eta / \epsilon$.

-(17) em nossa análise para a existência de conexão de órbitas ligando equilíbrios no plano $(\rho, m)$ e depois realizar um análise para a variável energia $E$. Para o equilíbrio em $\left(\rho_{m}, m_{m}\right)$ é possível mostrar que o equilíbrio é do tipo sela, de modo que a dimensão do espaço repulsor é 1. Para o equilíbrio em $\left(\rho_{r}, m_{r}\right)$ é possível mostrar que o ponto de equilíbrio é um atrator, gerando um espaço de dimensão 2. Observe, que neste caso o espaço de conexão ligando os equilíbrios será 3. Isto, tipicamente acontece, com choques do tipo Lax. A existência dessa conexão é conhecida e pode ser encontrada em [2], veja também a Figura 3.

Estamos interessados, entretanto, em analisar a existência de uma órbita conectando os estados $E_{r}$ até $E_{m}$. Observe, via equação (15), que o comportamento qualitativo da solução da EDO acompanhará, principalmente, o sinal do coeficiente multiplicando $E$. Ou seja, $-\frac{\eta / \epsilon+\beta \frac{d}{d \xi}(m / \rho)}{(-s+\beta(m / \rho))}$. Por outro lado, por meio da Figura 3 (e analiticamente) podemos observar que $m / \rho$ é constante em $\left(\rho_{m}, m_{m}\right)$ e depois é decrescente até conectar novamente o equilíbrio em $\left(\rho_{r}, m_{r}\right)$, então $\frac{d}{d \xi}(m / \rho)=0$ em $\left(\rho_{m}, m_{m}\right)$ e $\left(\rho_{r}, m_{r}\right) ;$ e $\frac{d}{d \xi}(m / \rho)<0$ para os valores em que $\rho$ e $m$ variam. O mesmo comportamento vale para $\frac{d}{d \xi}\left(m^{2} / \rho\right)$, ou seja, $\frac{d}{d \xi}\left(m^{2} / \rho\right)$ será zero nos equilíbrios e negativo no interior da região de conexã̃o.

Para o comportamento global da solução, temos duas análises a serem feitas. Para a primeira análise, estudamos o caso em que $s>\beta(m / \rho)$. Por meio de $(17)$, podemos ver que o comportamento depende fortemente da relação das escalas $\eta / \epsilon$ os quais podem ser subdivididas em:

(1) Razão $\eta / \epsilon$, tal que inicialmente tenhamos $d E / d \xi>0$. O sinal de $d E / d \xi$ só é positivo devido ao fato das variáveis $\rho$ e $m$, e suas derivadas, serem limitadas pois tais variáveis são solução do sistema (15)-(16). Dessa forma $E$ irá crescer até valores satisfazendo em que a derivada satisfaça $d E / d \xi=0$, o qual é um máximo. Tal máximo é limitado e é obtido quando a função energia $E$ iguala-se a uma relação entre as variáveis $\rho$ e $m$ através da seguinte relação:

$$
E=\left(a \frac{d}{d \xi}\left(\frac{m^{2}}{\rho}\right)-\frac{\epsilon}{\eta}\left(\frac{a^{2} \rho}{\gamma-1}+\frac{1}{2} \frac{m^{2}}{\rho}\right)\right)\left(\frac{\epsilon}{\eta}+\beta \frac{d}{d \xi}\left(\frac{m}{\rho}\right)\right)^{-1} .
$$

Note que $E$ é limitado e que se $\epsilon<<\eta$, ou seja, a relaxação ocorre numa escala menor que a escala da difusão, o pico praticamente não aparece; por outro lado se $\eta>>\epsilon$, ou seja, a difusão ocorre numa escala menor do que a relaxação, o pico é mais acentuado. Após o máximo da derivada, $d E / d \xi<0$ e portanto $E$ vai diminuindo. Observe que o sistema não atinge mais o equilíbrio, pois $d E / d \xi<0$ para os subsequentes valores de $E$, o qual tende a $-\infty$.

(2) Razão $\eta / \epsilon$, tal que inicialmente tenhamos $d E / d \xi<0$ desde o início. Por meio da EDO para $E$, podemos ver que neste caso não há pico e a solução diverge até $-\infty$.

Em ambos os casos acima não há órbita conectando equilíbrios. Se formos estudar mais cuidadosamente as características da Figura (2) percebemos que para valores pequenos de $\beta$, as 
características exibem, praticamente, um leque de rarefação após a curva iniciando-se em $x=0$ (note-se que isto não é uma rarefação). Apesar disso o formato da onda $E$ fica parecido ao de um perfil viajante, pois a relaxação atua muito fortémente e faz com que os valores dessa região tenda ao equilíbrio de modo bastante rápido. Mas note via Figura 2 que o choque atravessa as características da equação da energia $E$, influenciando a solução por um período de tempo longo não podendo assim exibir um perfil viajante.

Para o segundo caso, admitimos que $s<\beta(m / \rho)$. Então, pelo mesmo motivo afirmado anteriormente, temos que $d E / d \xi>0$. Neste caso a solução para energia $E$ irá crescer até atingir valores satisfazendo $d E / d \xi=0$, o qual é um máximo. Tal máximo é limitado e é obtido quando a relação (18) é satisfeita. Entretanto, note a partir da Equação (17) que até o momento em que $E$ satisfaz (18) este é um equilíbrio instável para a variável $E$. A partir desse momento temos $d E / d \xi<0$. Note, entretanto, que como $(-s+\beta(m / \rho))>0$, então a EDO é estável, ou seja, $E$

tende a valores limitados, quando $\xi \longrightarrow \infty, \frac{d}{d \xi}(m / \rho)$ e $\frac{d}{d \xi}\left(m^{2} / \rho\right)$ vão a zero e a solução tende ao equilíbrio. Dessa forma, existe a órbita conectando os estados.

Se olharmos para as características da Figura 2 percebemos que para valores grandes de $\beta$, as características estão muito próximas ao ponto de parecerem colidir (não colidem, devido ao teorema de existência e unicidade de equações diferenciais ordinárias, ver. e.g., [3]). Neste caso as características atravessam o choque de velocidade $s$ e a informação tem uma descontinuidade justamente nesta onda, fazendo com que o perfil de solução seja viajante, conforme solução do sistema (15)-(18), o que resumimos como:

Proposição 3.1. O sistema de Euler (1) com condições do tipo (2), e estados $\rho_{l}>\rho_{r}$ e $m_{l}>m_{r}$, exibe uma onda conectando $E_{m}$ e $E_{l}$ sendo um choque com perfil viscoso viajando com velocidade $s=\left(m_{m}-m_{l}\right) /\left(\rho_{m}-\rho_{r}\right)$, para $\beta>s(\rho / m)$. Tal onda é não monotônica, possuindo um máximo satisfazendo (18). Caso $\beta<s(\rho / m)$, a onda conectando tais estados não é uma onda viajante exibindo perfil viscoso. Além disso, a altura do pico depende exclusivamente da relação entre as escalas da difusão $\eta$ e da relaxação $\epsilon$.

\section{Referências}

[1] A. A. Bustos, Sistemas de leis de balanço em problemas de dinâmica de fluidos em meios porosos: modelagem matemática e aproximação numerica. Tese em Preparação.

[2] C. Dafermos, Hyperbolic Conservation Laws in Continuum Physics, Third Ed., Springer, 2009.

[3] F. John, Partial Differential Equations, Fourth Ed., Springer, c1982.

[4] R.J. Le Veque, Finite Volume Methods for Hyperbolic Problems, Cambridge Texts in applied Mathematics, UK, 2002.

[5] T-P Liu, Hyperbolic conservation laws with relaxation Communications in Mathematical Physics 108(1) 153-175 1987.

[6] P. Marcati, The One-Dimensional Darcy's Law as the Limit of a compressible Euler Flow, Journal of Differential Equations, 84 (1), 129-146, 1990. 